\title{
Formation Control of Second-Order Multiagent Systems with Time-Varying Delays
}

\author{
Hong Xia, Ting-Zhu Huang, Jin-Liang Shao, and Jun-Yan Yu \\ School of Mathematical Sciences, University of Electronic Science and Technology of China, Chengdu, Sichuan 611731, China \\ Correspondence should be addressed to Hong Xia; xiahong@uestc.edu.cn
}

Received 7 October 2013; Revised 16 December 2013; Accepted 16 December 2013; Published 8 January 2014

Academic Editor: Xiaojie Su

Copyright (C) 2014 Hong Xia et al. This is an open access article distributed under the Creative Commons Attribution License, which permits unrestricted use, distribution, and reproduction in any medium, provided the original work is properly cited.

\begin{abstract}
A formation control problem for second-order multiagent systems with time-varying delays is considered. First, a leader-following consensus protocol is proposed for theoretical preparation. With the help of Lyapunov-Krasovskii functional, a sufficient condition under this protocol is derived for stability of the multiagent systems. Then, the protocol is extended to the formation control based on a multiple leaders' architecture. It is shown that the agents will attain the expected formation. Finally, some simulations are provided to demonstrate the effectiveness of our theoretical results.
\end{abstract}

\section{Introduction}

Recent years have witnessed a rapidly growing interest in coordinated control of multiagent systems due to its broad applications in various disciplines [1-12]. As one of the important topics in this field, formation control has attracted great attention. Generally speaking, the main objective in formation control is to design appropriate protocol and algorithm such that the agents can achieve and preserve a predefined geometrical shape, such as a chain or a wedge. Potential applications of formation control include lots of cooperative tasks such as surveillance, exploration, search and rescue, transporting large objects, and control of arrays of satellites.

In the literature, researchers have proposed numerous approaches for the multiagent systems to achieve the anticipated formation, roughly categorized as behavior-based strategy [13], virtual structure method [14], and leader-following approach [15-21], to name a few. For instance, Xiao et al. [16] developed a formation framework of multiple leaders and applied a class of nonlinear consensus protocols to the formation control. Under the proposed framework, all the agents in the first-order systems could reach the expected formation. An adaptive formation control approach, in the absence of the velocity information of the leader, was proposed in [17]. Besides, the authors in [18] investigated the leader-following formation control problems for nonlinear systems under fixed and switching topologies. The above works, however, did not take into account the effects of time delays.

Owing to the finite speed of information transmission and processing, time delays are inevitable in multiagent systems. In particular, one type of time delays is communication delays, whose effects on multiagent systems have been addressed by many researchers [3-9]. For formation control with time delays, Luo et al. [19] gave a sufficient condition of formation control of multiagent systems by using Lyapunov stability theory. Also, Rezaee and Abdollahi [20] provided a motion synchronization strategy with time delays. Note that the time delays in both papers were assumed to be constant. In reality, it is more practical when the timevarying delays are accounted for. Lu et al. [21] studied the formation control of second-order multiagent systems with time-varying delays, where the time delays existed only in the transmission of position information between neighbors. For second-order multiagent systems, it is worthwhile to mention that the information exchanged between neighbors may include velocity information as well as position information.

Motivated by the above analysis, we consider a leaderfollowing formation control problem for second-order multiagent systems, with time-varying delays existing in the transmission of both velocity and position. Here, we adopt the 
formation framework proposed in [16]. More specifically, the formation information is divided into two parts: the global one and the local one, where the former determines the geometric pattern of the desired formation and the latter decides the relative information of agents with respect to their neighbors. Only a small number of agents called leaders have access to the global information, and the other agents called followers regulate their states according to the local information.

An outline of this paper is as follows. Section 2 provides some preliminary notions of graph theory and formulates the formation control problem. By utilizing Lyapunov-Krasovskii functional, Section 3 presents the main results under the proposed control protocol. Then several simulations are illustrated in Section 4. Finally, the conclusion is drawn in Section 5.

The following notations will be used throughout this paper. Given a matrix, the superscripts " $T$ " and " -1 " stand for its transposition and inverse, respectively; $\Lambda(\cdot)$ and $\|\cdot\|$ denote the set of all eigenvalues and the spectral norm of the matrix, respectively. Let $I_{n}$ be an $n \times n$ identity matrix, $\mathbf{1}_{n}=[1 \cdots 1]^{T} \in \mathbb{R}^{n}$, and $0_{n \times n}$ represents an $n \times n$ zero matrix. And $\operatorname{diag}\left\{b_{1}, \ldots, b_{n}\right\}$ denotes a diagonal matrix with diagonal elements being $b_{1}, \ldots, b_{n}$. For a complex number $\mu \in \mathbb{C}$, $\operatorname{Re}(\mu), \operatorname{Im}(\mu)$, and $|\mu|$ are its real part, imaginary part, and modulus, respectively. $\otimes$ denotes the Kronecker product.

\section{Preliminaries and Problem Formulation}

Let $\mathscr{G}=(\mathscr{V}, \mathscr{E}, A)$ be a weighted directed graph with the set of nodes $\mathscr{V}=\left\{v_{1}, v_{2}, \ldots, v_{n}\right\}$, set of edges $\mathscr{E} \subseteq \mathscr{V} \times \mathscr{V}$, and a weighted adjacency matrix $A=\left[a_{i j}\right]$ with nonnegative elements $a_{i j}$. The node indexes belong to a finite index set $\mathscr{I}=\{1,2, \ldots, n\}$. An edge in $\mathscr{E}$ is denoted by $e_{j i}=\left(v_{j}, v_{i}\right)$, where $e_{j i} \in \mathscr{E}$ if and only if $a_{i j} \neq 0$. In this case, we say node $j$ is a neighbor of node $i$ and denote the neighbors of node $i$ by $\mathcal{N}_{i}=\left\{j \in \mathscr{V}:\left(v_{j}, v_{i}\right) \in \mathscr{E}\right\}$. Moreover, we assume $a_{i i}=0$ for all $i \in \mathscr{V}$. Let the Laplacian matrix $L=\left[l_{i j}\right] \in \mathbb{R}^{n \times n}$ associated with $A$ be defined as $l_{i i}=\sum_{j=1, j \neq i}^{n} a_{i j}$ and $l_{i j}=-a_{i j}$.

A directed path in directed graph $\mathscr{G}$ from $v_{i_{1}}$ to $v_{i_{k}}$ is a sequence of edges of the form $\left(v_{i_{1}}, v_{i_{2}}\right),\left(v_{i_{2}}, v_{i_{3}}\right), \ldots,\left(v_{i_{k-1}}, v_{i_{k}}\right)$, where $v_{i_{j}} \in \mathscr{V}$ for $j=1,2, \ldots, k$. A directed graph is called strongly connected if any two distinct nodes of the graph can be connected via a directed path that follows the edges of the graph. A directed tree is a directed graph, where every node, except one special node without any parent, which is called the root, has exactly one parent, and the root can be connected to any other nodes through paths. A spanning tree of a digraph is a directed tree formed by graph edges that connect all the nodes of the graph.

For the $n$-agent system considered in this paper, suppose that there are $m(m \leq n)$ leaders and $n-m$ followers. For convenience, we use $\mathscr{R}=\{1,2, \ldots, m\}$ and $\mathscr{F}=\{m+$ $1, m+2, \ldots, n\}$ to denote, respectively, the leader set and the follower set. Further, the interaction topology among agents is modeled by a direct graph $\mathscr{G}$.

The dynamics of the $n$ autonomous agents are given by

$$
\dot{x}_{i}=v_{i}, \quad \dot{v}_{i}=u_{i}, \quad i=1,2, \ldots, n,
$$

where $x_{i}, v_{i}, u_{i} \in \mathbb{R}^{p}$ denote the position, velocity and control input of agent $i$, respectively.

Using the formation framework in [16], the formation information is divided into two independent parts: the global one and the local one. The global information, which determines the geometric pattern of the expected formation, is represented by a time-dependent column vector $F=\left[f_{1}^{T}, f_{2}^{T}, \ldots, f_{m}^{T}\right]^{T} \in \mathbb{R}^{2 m p}$ with $f_{i}=\left[f_{i}^{x^{T}}, f_{i}^{v^{T}}\right]^{T} \in$ $\mathbb{R}^{2 p}(i \in \mathscr{R})$. The local formation information is denoted by a time-independent nonnegative matrix $W=\left[W_{m+1}^{T}, \ldots, W_{n}^{T}\right]^{T} \in \mathbb{R}^{(n-m) \times m}$ with unit entry sum for each row $W_{i}=\left(w_{i}^{1}, \ldots, w_{i}^{m}\right) \in \mathbb{R}^{1 \times m}(i \in \mathscr{F})$.

Denote $x^{\mathscr{R}}=\left(x_{1}^{T}, x_{2}^{T}, \ldots, x_{m}^{T}\right)^{T}$ and $v^{\mathscr{R}}=\left(v_{1}^{T}, v_{2}^{T}, \ldots\right.$, $\left.v_{m}^{T}\right)^{T}$; we have the following.

Definition 1. We say that system (1) solves the formation problem if there exists a $\mathbb{R}^{2 p}$-valued function $f_{c}=$ $\left[f_{c}^{x}(t)^{T}, f_{c}^{v}(t)^{T}\right]$ with $f_{c}^{x}(t) \in \mathbb{R}^{p}, f_{c}^{v}(t) \in \mathbb{R}^{p}$, and $\dot{f}_{c}^{x}(t)=$ $f_{c}^{v}(t)$ such that $x_{i} \rightarrow f_{i}^{x}(t)+f_{c}^{x}(t), v_{i} \rightarrow f_{i}^{v}(t)+f_{c}^{v}(t)$ for $i \in \mathscr{R}$ and $x_{i} \rightarrow\left(W_{i} \otimes I_{p}\right) x^{\mathscr{R}}, v_{i} \rightarrow\left(W_{i} \otimes I_{p}\right) v^{\mathscr{R}}$ for $i \in \mathscr{F}$ as $t \rightarrow \infty$. In particular, the formation problem is called a timeinvariant formation (TIF) problem, a time-varying formation (TVF) problem, and a time-varying formation for trajectory tracking (TVFT) problem if $\dot{f}_{i}^{x}=\dot{f}_{c}^{x}=f_{i}^{v}=f_{c}^{v}=0$, $\dot{f}_{i}^{x}=f_{i}^{v} \neq 0$ with $\dot{f}_{c}^{x}=f_{c}^{v}=0$ and $\dot{f}_{i}^{x}=f_{i}^{v} \neq 0$ with $\dot{f}_{c}^{x}=f_{c}^{v} \neq 0$, respectively.

The column vector $f_{c}(t)$, which specifies the state of the formation and may be dependent on initial states or may be an external input, used to guide the group of agents to track a prescribed trajectory. The vector $F$ defines the basic frame of the anticipated formation formed by the leaders, and the nonnegative matrix $W$ specifies the local-state restrictions of followers with respect to their leader neighbors. Since each row entry sum of $W$ equals 1 , the followers should lie in the convex region covered by the leaders.

\section{Main Results}

3.1. Leader-Following Consensus. For the better understanding of the formation control problem, we first consider a leader-following consensus problem. The multiagent systems solve a leader-following consensus asymptotically if $\lim _{t \rightarrow \infty}\left\|x_{i}-f_{0}^{x}\right\|=0$ and $\lim _{t \rightarrow \infty}\left\|v_{i}-f_{0}^{v}\right\|=0$ for all $i=$ $1,2, \ldots, n$. Under this circumstance, all the agents can obtain the acceleration $\dot{f}_{0}^{v}$, but only the root agent can obtain the difference between its state and the formation information, and hence it can pin the other leaders to attain the anticipated formation. Define a matrix $B \in \mathbb{R}^{n \times n}$ as $B=\operatorname{diag}\left\{b_{1}, \ldots, b_{n}\right\}$, where $b_{i}>0$ if the interaction topology $\mathscr{G}$ has a spanning tree rooted at $i, 1 \leq i \leq n$ and $b_{i}=0$ otherwise.

The time-varying delay $\tau(t)$ is the time delay for information communicated between agents at time $t$. Owing to the communication time delays, each agent cannot instantly get the information from others. With time-varying delays 
existing in the transmission of both velocity and position, we now provide the following consensus protocol:

$$
\begin{array}{r}
u_{i}(t) \\
=\dot{f}_{0}^{v}+\sum_{j \in \mathcal{N}_{i}} a_{i j}\left[\left(x_{j}(t-\tau(t))-x_{i}(t-\tau(t))\right)\right. \\
\left.+k\left(v_{j}(t-\tau(t))-v_{i}(t-\tau(t))\right)\right] \\
+b_{i}\left[\left(f_{0}^{x}(t-\tau(t))-x_{i}(t-\tau(t))\right)\right. \\
\left.+k\left(f_{0}^{v}(t-\tau(t))-v_{i}(t-\tau(t))\right)\right], \\
i=1,2, \ldots, n,
\end{array}
$$

where the control parameter $k>0, A=\left(a_{i j}\right)_{n \times n}$ is the adjacency matrix corresponding to the graph $\mathscr{G}, b_{i}>0$ if the interaction topology $\mathscr{G}$ has a spanning tree rooted at $i$ and $b_{i}=0$ otherwise, and the time-varying delay $\tau(t)$ is a continuously differentiable function with

$$
\tau(t)<d_{1}, \quad \dot{\tau}(t) \leq d_{2}<1 .
$$

Denote $x=\left(x_{1}^{T}, x_{2}^{T}, \ldots, x_{n}^{T}\right)^{T}, v=\left(v_{1}^{T}, v_{2}^{T}, \ldots, v_{n}^{T}\right)^{T}$; then, system (1) with protocol (2) can be written in a matrix form:

$$
\begin{gathered}
\dot{x}=v, \\
\dot{v}=\dot{f}_{0}^{v} \mathbf{1}_{n}-(L+B) \otimes I_{p} x(t-\tau) \\
-k(L+B) \otimes I_{p} v(t-\tau) \\
+B\left[f_{0}^{x}(t-\tau) \cdot \mathbf{1}_{n}+k f_{0}^{v}(t-\tau) \cdot \mathbf{1}_{n}\right],
\end{gathered}
$$

where $L$ is the Laplacian matrix associated with $\mathscr{G}, B=$ $\operatorname{diag}\left\{b_{1}, \ldots, b_{n}\right\}, b_{i}>0$ if the interaction topology $\mathscr{G}$ has a spanning tree rooted at $i$ and $b_{i}=0$ otherwise.

Let $\bar{x}=x-f_{0}^{x} \mathbf{1}_{n}, \bar{v}=v-f_{0}^{v} \mathbf{1}_{n}$; then, (4) can be rewritten as

$$
\begin{gathered}
\dot{\bar{x}}=\bar{v} \\
\dot{\bar{v}}=-(L+B) \otimes I_{p} \bar{x}(t-\tau)-k(L+B) \otimes I_{p} \bar{v}(t-\tau) .
\end{gathered}
$$

Taking $\varepsilon=\left(\bar{x}^{T}, \bar{v}^{T}\right)^{T},(5)$ can be equally expressed as

$$
\dot{\varepsilon}=Y \varepsilon(t)+Z \varepsilon(t-\tau),
$$

where

$$
\begin{gathered}
Y=\left(\begin{array}{cc}
0_{n \times n} & I_{n} \\
0_{n \times n} & 0_{n \times n}
\end{array}\right) \otimes I_{p}, \\
Z=\left(\begin{array}{cc}
0_{n \times n} & 0_{n \times n} \\
-H & -k H
\end{array}\right) \otimes I_{p}, \\
H=L+B .
\end{gathered}
$$

To proceed further, we need the following lemmas.

Lemma 2 (see [22]). Given a complex-coefficient polynomial

$$
r(z)=z^{2}+(a+i b) z+c+i d,
$$

where $a, b, c, d \in \mathbb{R}, r(z)$ is Hurwitz stable if and only if $a>0$ and $a b d+a^{2} c-d^{2}>0$.
Lemma 3. The matrix $F=Y+Z=\left(\begin{array}{cc}0_{n \times n} & I_{n} \\ -H & -k H\end{array}\right) \otimes I_{p}$ is Hurwitz stable if and only if $H$ is positive stable and $k>$ $\max _{\lambda \in \Lambda(H)}(|\operatorname{Im}(\lambda)| /(\sqrt{|\operatorname{Re}(\lambda)|} \cdot|\lambda|))$.

Proof. Let $z$ be an eigenvalue of $F$. Then one has

$$
\begin{aligned}
\operatorname{det}\left(z I_{2 n p}-F\right) & =\operatorname{det}\left(\left[\begin{array}{cc}
z I_{n} & -I_{n} \\
H & z I_{n}+k H
\end{array}\right] \otimes I_{p}\right) \\
& =\operatorname{det}\left(\left(z\left(z I_{n}+k H\right)+H\right) \otimes I_{p}\right) \\
& =\prod_{i=1}^{n}\left(z^{2}+k \lambda_{i} z+\lambda_{i}\right)^{p} .
\end{aligned}
$$

Clearly, the Hurwitz stability of matrix $F$ is equivalent to that of the polynomial: $r(z)=z^{2}+k \lambda_{i} z+\lambda_{i}$, where $\lambda_{i}=\operatorname{Re}\left(\lambda_{i}\right)+$ $i \operatorname{Im}\left(\lambda_{i}\right)$ is the $i$ th eigenvalue of $H$. It follows from Lemma 2 that $r(z)$ is Hurwitz stable if and only if $\operatorname{Re}\left(\lambda_{i}\right)>0$ and $k>$ $\left|\operatorname{Im}\left(\lambda_{i}\right)\right| /\left(\sqrt{\left|\operatorname{Re}\left(\lambda_{i}\right)\right|} \cdot\left|\lambda_{i}\right|\right), \lambda_{i} \in \Lambda(H)$.

Lemma 4 (see [4]). The matrix $H=L+B$ is positive stable if $G$ has a spanning tree.

Now we give the main result of this subsection.

Theorem 5. For system (6), take

$$
k>k_{0}=\max _{\lambda \in \Lambda(H)} \frac{|\operatorname{Im}(\lambda)|}{\sqrt{|\operatorname{Re}(\lambda)|} \cdot|\lambda|}
$$

and suppose that

$$
0<\tau<\tau_{0}=\frac{1}{2\left\|Z^{T} Z\right\| /\left(1-d_{2}\right)+\left\|2 Y^{T} Y+P Z Z^{T} P\right\|} .
$$

Then, the consensus is reached asymptotically if the graph $\mathscr{G}$ has a spanning tree.

Proof. Since $\mathscr{G}$ has a spanning tree, $H$ is positive stable based on Lemma 4. It follows from Lemma 3 that $F$ is Hurwitz stable. Therefore, there exists a positive definite matrix $P \in$ $\mathbb{R}^{2 n p \times 2 n p}$ such that

$$
P F+F^{T} P=-I_{2 n p}
$$

To analyze the convergence of system (6), we define a Lyapunov-Krasovskii functional

$$
\begin{aligned}
V(\varepsilon)= & \varepsilon^{T}(t) P \varepsilon(t)+\int_{t-\tau}^{T} \varepsilon^{T}(t) S \varepsilon(t) d s \\
& +\int_{-\tau}^{0} \int_{t+\theta}^{t} \dot{\varepsilon}^{T}(s) R \dot{\varepsilon}(s) d s d \theta,
\end{aligned}
$$

where $S=\beta I_{2 n p}$ and $R=I_{2 n p}$.

Calculating $\dot{V}(\varepsilon)$ along the solution of (6), we have

$$
\begin{aligned}
\dot{V}(\varepsilon)= & \varepsilon^{T}(t)\left(Y^{T} P+P Y\right) \varepsilon(t)+2 \varepsilon^{T}(t) P Z \varepsilon(t-\tau) \\
& +\varepsilon^{T}(t) \beta \varepsilon(t)-\beta(1-\dot{\tau}) \varepsilon^{T}(t-\tau) \varepsilon(t-\tau) \\
& -\int_{t-\tau}^{t} \dot{\varepsilon}^{T}(\theta) \dot{\varepsilon}(\theta) d \theta+\tau \dot{\varepsilon}^{T}(t) \dot{\varepsilon}(t) .
\end{aligned}
$$


Due to the fact that $\varepsilon(t-\tau)=\varepsilon(t)-\int_{t-\tau}^{t} \dot{\varepsilon}(s) d s$ and $2 a^{T} b \leq a^{T} \Psi^{-1} a+b^{T} \Psi b$ holds for any appropriate positive definite matrix $\Psi$, we can obtain that

$$
\begin{aligned}
2 \varepsilon^{T}(t) & P Z \varepsilon(t-\tau) \\
= & \varepsilon^{T}(t)\left(Z^{T} P+P Z\right) \varepsilon(t)+2 \int_{t-\tau}^{t}\left(-Z^{T} P \varepsilon(t)\right)^{T} \dot{\varepsilon}(s) d s \\
\leq & \varepsilon^{T}(t)\left(Z^{T} P+P Z\right) \varepsilon(t)+\tau \varepsilon^{T}(t) P Z Z^{T} P \varepsilon(t) \\
& \quad+\int_{t-\tau}^{t} \dot{\varepsilon}^{T}(s) \dot{\varepsilon}(s) d s .
\end{aligned}
$$

Similarly,

$$
\begin{aligned}
\tau \dot{\varepsilon}^{T}(t) \dot{\varepsilon}(t)= & \tau[Y \varepsilon(t)+Z \varepsilon(t-\tau)]^{T}[Y \varepsilon(t)+Z \varepsilon(t-\tau)] \\
= & \tau\left[\varepsilon^{T}(t) Y^{T} Y \varepsilon(t)+\varepsilon^{T}(t-\tau) Z^{T} Z \varepsilon(t-\tau)\right] \\
& +\varepsilon^{T}(t) Y^{T} Z \varepsilon(t-\tau)+\varepsilon^{T}(t-\tau) Z^{T} Y \varepsilon(t) \\
\leq & 2 \tau\left[\varepsilon^{T}(t) Y^{T} Y \varepsilon(t)+\varepsilon^{T}(t-\tau) Z^{T} Z \varepsilon(t-\tau)\right] .
\end{aligned}
$$

Substituting (15) and (16) into (14) leads to

$$
\begin{aligned}
\dot{V}(\varepsilon) \leq & \varepsilon^{T}(t)\left[-\left(P F+F^{T} P\right)+\beta I_{2 n p}\right. \\
& \left.+\tau\left(2 Y^{T} Y+P Z Z^{T} P\right)\right] \varepsilon(t) \\
& +\varepsilon^{T}(t-\tau)\left[-(1-\dot{\tau}) \beta I_{2 n p}+2 \tau Z^{T} Z\right] \varepsilon(t-\tau) \\
\leq & \varepsilon^{T}(t)\left(-1+\beta+\tau\left\|2 Y^{T} Y+P Z Z^{T} P\right\|\right) \varepsilon(t) \\
& +\varepsilon^{T}(t-\tau)\left[-\left(1-d_{2}\right) \beta+2 \tau\left\|Z^{T} Z\right\|\right] \varepsilon(t-\tau) .
\end{aligned}
$$

Consequently, a sufficient condition for $\dot{V}(\varepsilon)<0$ is

$$
\begin{gathered}
-1+\beta+\tau\left\|2 Y^{T} Y+P Z Z^{T} P\right\|<0, \\
-\left(1-d_{2}\right) \beta+2 \tau\left\|Z^{T} Z\right\|<0 .
\end{gathered}
$$

From (18), we can obtain that

$$
\tau<\tau_{0}=\frac{1}{2\left\|Z^{T} Z\right\| /\left(1-d_{2}\right)+\left\|2 Y^{T} Y+P Z Z^{T} P\right\|} .
$$

Therefore, by Lyapunov-Krasovskii Theorem (see [23]), the error system (6) is uniformly asymptotically stable. Namely, the consensus is reached asymptotically.

Remark 6. It can be seen that many zoom techniques are applied during the derivation of $\tau_{0}$, which result in a conservative estimation of $\tau_{0}$.
3.2. Time-Varying Formation for Trajectory Tracking. In this subsection, we consider the case that the agents form a time-varying formation as they track the desired trajectory. More specifically, the desired trajectory of the formation, represented by $f_{c}=\left[f_{c}^{x}(t)^{T}, f_{c}^{v}(t)^{T}\right]$, is assumed to be determined by the following equation:

$$
\dot{f}_{c}^{x}=f_{c}^{v}=g\left(t, f_{c}^{x}\right) .
$$

Also make the following assumptions about the multiagent system:

(A1) the local interaction topology of the leaders has a spanning tree, and the leaders' dynamic is unaffected by the followers;

(A2) the root agent in the local interaction topology of the leaders is able to access the reference trajectory;

(A3) in addition to the local information from their neighbors, the leaders can also obtain the global information $F$;

(A4) in addition to the local information from their follower neighbors, each follower can also get the local formation $W$ directly or indirectly from the leaders.

Using the following control protocol:

$$
\begin{gathered}
\dot{x}_{i}=v_{i}, \quad i=1,2, \ldots, n, \\
\dot{v}_{i}=\dot{f}_{i}^{v}+\dot{g}\left(t, f_{c}^{x}\right) \\
+\sum_{j=1}^{m} a_{i j}\left\{\left[\left(x_{j}(t-\tau)-f_{j}^{x}(t-\tau)\right)\right.\right. \\
\left.-\left(x_{i}(t-\tau)-f_{i}^{x}(t-\tau)\right)\right] \\
+k\left[\left(v_{j}(t-\tau)-f_{j}^{v}(t-\tau)\right)\right. \\
\left.\left.-\left(v_{i}(t-\tau)-f_{i}^{v}(t-\tau)\right)\right]\right\} \\
-b_{i}\left[\left(x_{i}(t-\tau)-f_{i}^{x}(t-\tau)-f_{c}^{x}(t-\tau)\right)\right. \\
\left.+k\left(v_{i}(t-\tau)-f_{i}^{v}(t-\tau)-g(t-\tau)\right)\right],
\end{gathered}
$$$$
\dot{v}_{i}=\sum_{k=1}^{m} w_{i}^{k} \dot{v}_{k}
$$

$$
\begin{aligned}
+\sum_{j=1}^{n} a_{i j}\left\{\left[\left(x_{j}(t-\tau)-\sum_{k=1}^{m} w_{j}^{k} x_{k}(t-\tau)\right)\right.\right. \\
\left.-\left(x_{i}(t-\tau)-\sum_{k=1}^{m} w_{i}^{k} x_{k}(t-\tau)\right)\right] \\
+k\left[\left(v_{j}(t-\tau)-\sum_{k=1}^{m} w_{j}^{k} v_{k}(t-\tau)\right)\right. \\
\left.\left.-\left(v_{i}(t-\tau)-\sum_{k=1}^{m} w_{i}^{k} v_{k}(t-\tau)\right)\right]\right\}
\end{aligned}
$$




$$
\begin{array}{r}
-b_{i}\left[\left(x_{i}(t-\tau)-\sum_{k=1}^{m} w_{i}^{k} x_{k}(t-\tau)\right)\right. \\
\left.+k\left(v_{i}(t-\tau)-\sum_{k=1}^{m} w_{i}^{k} v_{k}(t-\tau)\right)\right], \\
i \in \mathscr{F},
\end{array}
$$

where $k>0, A=\left(a_{i j}\right)_{n \times n}$ is the adjacency matrix corresponding to the graph $\mathscr{G}$, and $b_{i}>0$ if there is a spanning tree rooted at $i$ in the graph $\mathscr{G}$ and $b_{i}=0$ otherwise, we have the following result.

Theorem 7. Suppose that the graph $\mathscr{G}$ has a spanning tree. Take

$$
\begin{gathered}
k>k_{0}=\max _{\lambda \in \Lambda(H)} \frac{|\operatorname{Im}(\lambda)|}{\sqrt{|\operatorname{Re}(\lambda)|} \cdot|\lambda|}, \\
0<\tau<\tau_{0}=\frac{1}{2\left\|Z^{T} Z\right\| /\left(1-d_{2}\right)+\left\|2 Y^{T} Y+P Z Z^{T} P\right\|} .
\end{gathered}
$$

With protocol (21), the multiagent systems attain a timevarying formation for trajectory tracking (TVFT) under assumptions (A1)-(A4).

Proof. Let $\widetilde{x}_{i}=x_{i}-f_{i}^{x}-f_{c}^{x}, \widetilde{v}_{i}=v_{i}-f_{i}^{v}-g\left(t, f_{c}^{x}\right)$ for $i \in \mathscr{R}$, and $\widetilde{x}_{i}=x_{i}-\sum_{k=1}^{m} w_{i}^{k} x_{k}, \widetilde{v}_{i}=v_{i}-\sum_{k=1}^{m} w_{i}^{k} v_{k}$ for $i \in \mathscr{F}$. Then we can rewrite protocol (21) as

$$
\begin{gathered}
\dot{\tilde{x}}_{i}=\widetilde{v}_{i}, \\
\dot{\tilde{v}}_{i}=\sum_{j=1}^{n} a_{i j}\left[\left(\tilde{x}_{j}(t-\tau)-\tilde{x}_{i}(t-\tau)\right)\right. \\
\left.+k\left(\widetilde{v}_{j}(t-\tau)-\widetilde{v}_{i}(t-\tau)\right)\right] \\
-b_{i}\left(\tilde{x}_{i}(t-\tau)+k \widetilde{v}_{i}(t-\tau)\right), \quad i=1,2, \ldots, n .
\end{gathered}
$$

Denote $\widetilde{x}=\left(\widetilde{x}_{1}^{T}, \widetilde{x}_{2}^{T}, \ldots, \widetilde{x}_{n}^{T}\right)$ and $\widetilde{v}=\left(\widetilde{v}_{1}^{T}, \widetilde{v}_{2}^{T}, \ldots, \widetilde{v}_{n}^{T}\right) ;(23) \mathrm{can}$ be expressed in a matrix form:

$$
\begin{gathered}
\dot{\tilde{x}}=\widetilde{v}, \\
\dot{\tilde{v}}=-(L+B) \otimes I_{p} \widetilde{x}(t-\tau)-k(L+B) \otimes I_{p} \widetilde{v}(t-\tau) .
\end{gathered}
$$

It follows from Theorem 5 that $\widetilde{x}_{i}$ and $\widetilde{v}_{i}$ converge to zero asymptotically, and equally, $x_{i} \rightarrow f_{i}^{x}+f_{c}^{x}, v_{i} \rightarrow f_{i}^{v}+f_{c}^{v}$ for $i \in \mathscr{R}$ and $x_{i} \rightarrow\left(W_{i} \otimes I_{p}\right) x^{\mathscr{R}}, v_{i} \rightarrow\left(W_{i} \otimes I_{p}\right) v^{\mathscr{R}}$ for $i \in \mathscr{F}$ as $t \rightarrow \infty$.

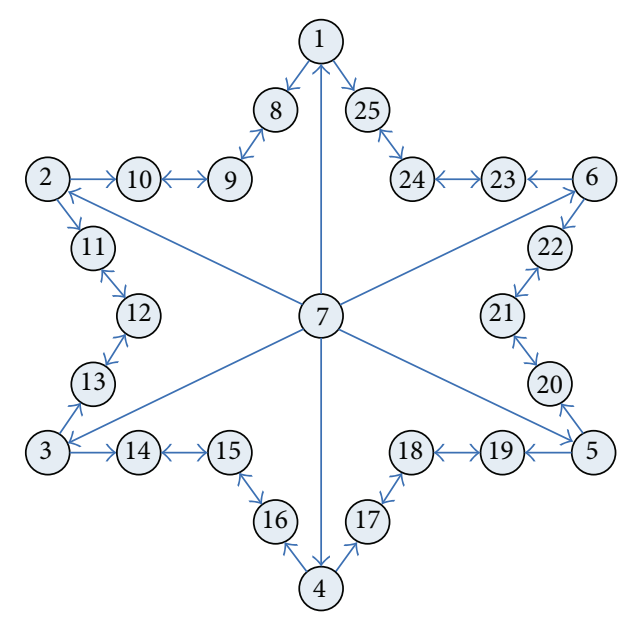

Figure 1: The interaction topology.

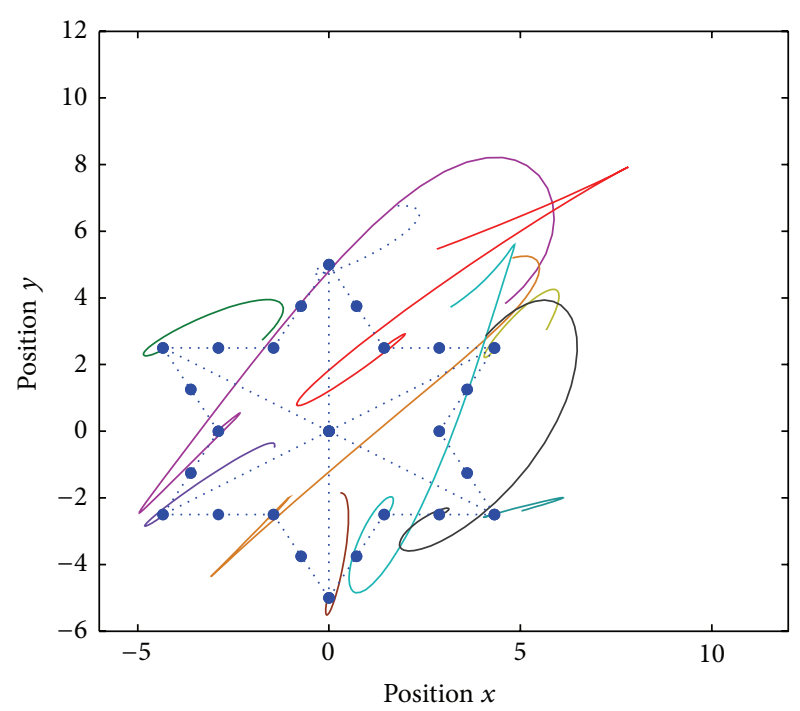

FIgURE 2: The trajectories of some agents in TIF.

Remark 8. Obviously, Theorem 7 still holds if $\dot{f}_{i}^{x}=f_{i}^{v}=0$, or $\dot{f}_{i}^{x}=f_{i}^{v} \neq 0$ and $\dot{f}_{c}^{x}=f_{c}^{v}=g\left(t, f_{c}^{x}\right)=0$. In the form case, the multiagent systems attain a time-invariant formation (TIF). In the latter case, the multiagent systems attain a time-varying formation.

\section{Simulations}

In this section, to illustrate our theoretical results derived in the above section, we will provide several examples. Consider a multiagent system consisting of 25 agents moving in a plane (i.e., $p=2$ ), with the direct interaction topology described in Figure 1 . The expected formation is a hexagram. Assume that the first 7 agents are leaders with the spanning tree rooted at agent 7. For simplicity, let $a_{i j}=1$ if agent $j$ is a neighbor of agent $i$ and $a_{i j}=0$ otherwise. With simple calculations, we can obtain that $k_{0}=0$ and $\tau_{0}=0.007$. Take $k=2, \tau=$ $0.0065|\cos t|$, and $d_{2}=0.05$. 


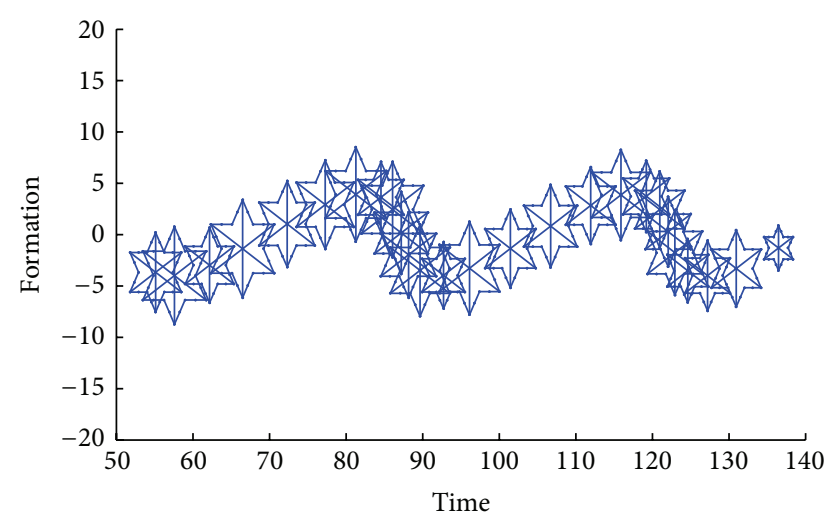

FIgURE 3: The formation states of agents in TVFT.

Consider

$$
\begin{gathered}
f_{i}^{x}=R_{i}(t)\left(\begin{array}{c}
\cos \left(\frac{\pi}{2}+\frac{2(i-1)}{3} \pi\right) \\
\sin \left(\frac{\pi}{2}+\frac{2(i-1)}{3} \pi\right)
\end{array}\right), \quad i=1,2, \ldots, 6, \\
R_{i}(t) \equiv 5(\mathrm{TIF}), \\
f_{i}^{x}=(0,0)^{T}, \quad i=7, \\
\dot{f}_{i}^{x}=f_{i}^{v}, \quad i=1,2, \ldots, 25, \\
f_{c}^{x}=4\left(\begin{array}{l}
\sin 3 t \\
\sin 3 t
\end{array}\right), \quad f_{c}^{v}=\dot{f}_{c}^{x}, \\
R_{i}(t)=6 \sin t(\mathrm{TVFT}), \\
w_{24}^{1}=w_{24}^{6}=w_{24}^{7}=w_{3 i}^{i-1}=w_{3 i}^{i-2}=w_{3 i}^{7}=\frac{1}{3}, \\
w_{i}^{j}=0, \quad i=3,4, \ldots, 7, \\
w_{8}^{1}=w_{25}^{1}=w_{3 i+1}^{i-1}=w_{3 i+2}^{i-1}=\frac{2}{3}, \quad i=3,4, \ldots, 7, \\
w_{3 i+1}^{2}=w_{3 i+2}^{i-2}=w_{3 i-1}^{7}=w_{3 i+1}^{7}=\frac{1}{6}, \quad i=3,4, \ldots, 6 \\
w_{3 i-1}^{7}=w_{3 i+1}^{7}=\frac{1}{6}, \quad i=7,8, \\
w_{3}^{7},
\end{gathered}
$$

Figure 2 shows the trajectories of some agents in timeinvariant formation (TIF), where the initial states of the agents are randomly generated in a given bounded region. From Figure 2, we can see that the agents attain the vertexes of a hexagram. In other words, the expected time-invariant formation is reached. For the trajectory tracking formation, Figure 3 illustrates the formation states of agents. Since the expected trajectory of the formation $f_{c}^{x}$ satisfies the equation in (25), the formation in Figure 3 changes with time as it moves along a sinusoidal curve. Furthermore, the position and velocity errors of agents in TVFT with time delays

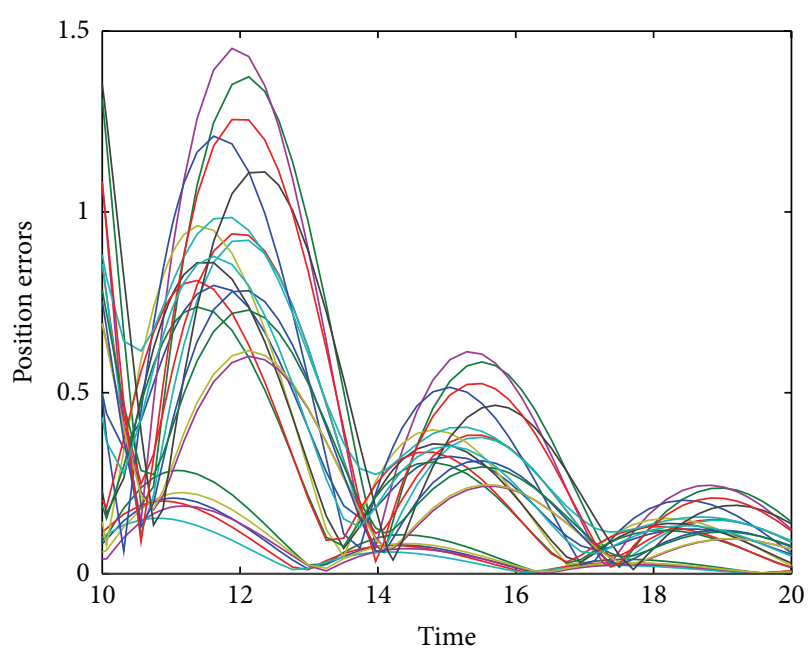

(a) Without velocity transmission delays

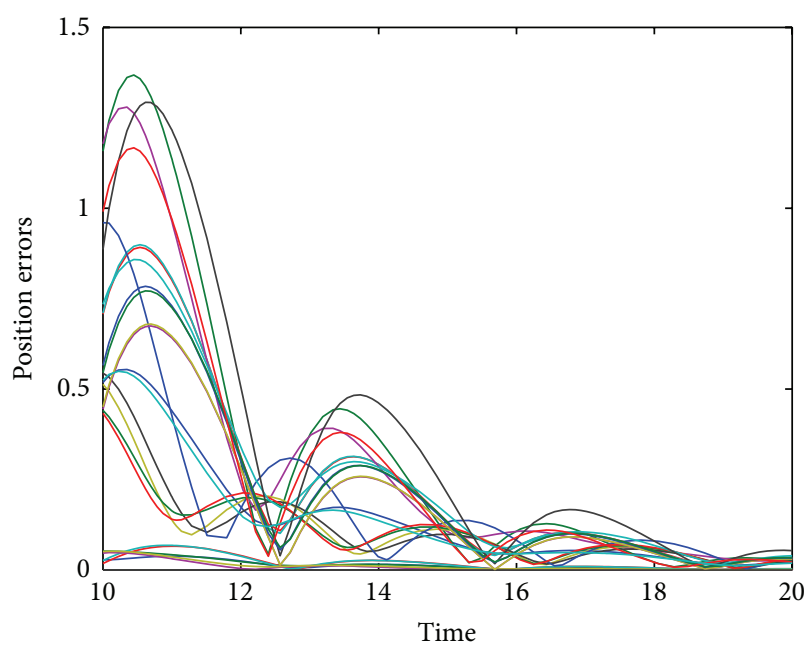

(b) With velocity transmission delays

FIGURE 4: The position errors of agents in TVFT.

existing only in the transmission of position are shown in Figures 4(a) and 5(a), respectively, and the position and velocity errors of agents in TVFT with time delays existing in the transmission of both position and velocity are shown in Figures 4(b) and 5(b), respectively. It can be seen that all of the errors converge to zero ultimately, while the errors in the latter figures converge to zero faster than those in the former figures.

\section{Conclusion}

This paper investigates the formation control problem for second-order multiagent systems with time-varying delays. We first consider a leader-following consensus problem. By employing Lyapunov-Krasovskii functional, we prove that the multiagent systems can reach consensus. Then, under a special multiple leaders' framework, we apply the protocol to the formation control, and derive a sufficient condition for the system to achieve prescribed formation. Moreover, several 


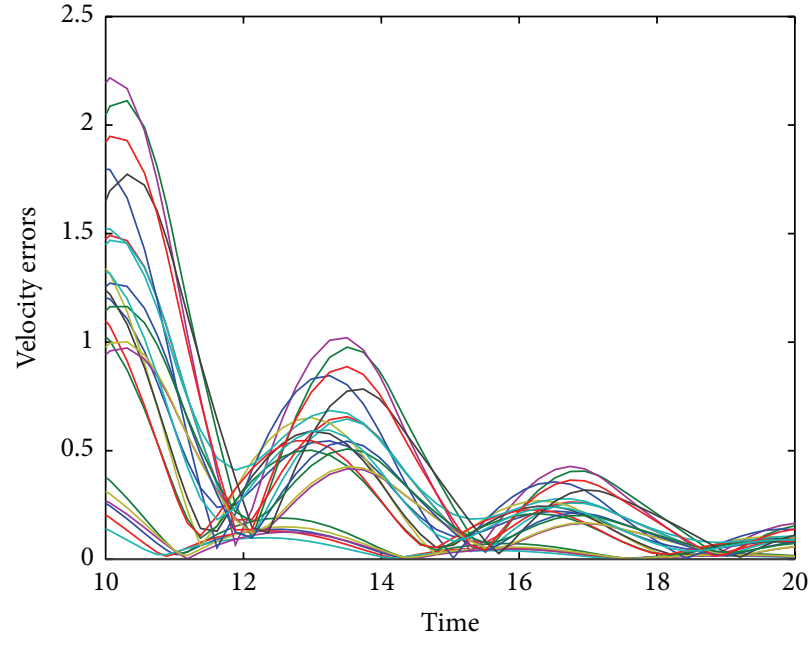

(a) Without velocity transmission delays

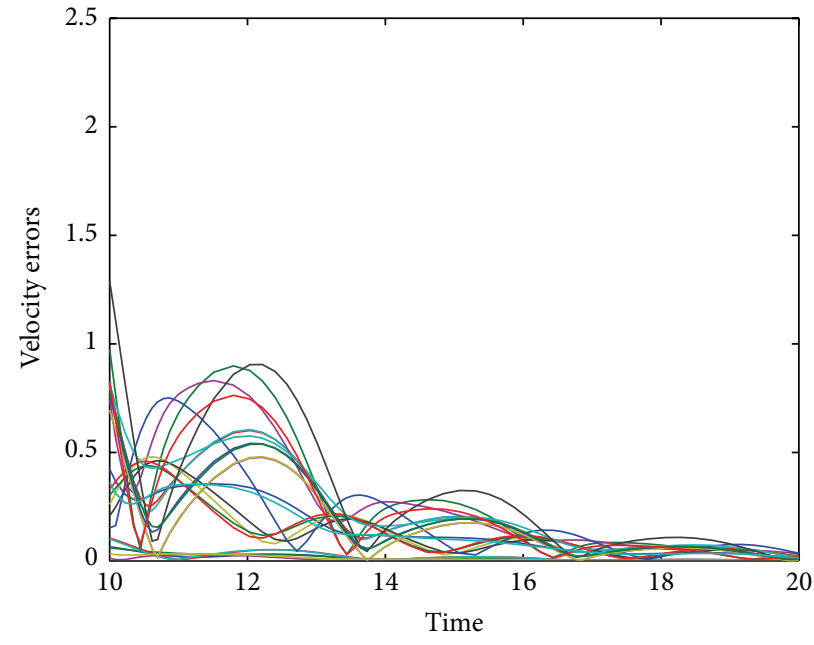

(b) With velocity transmission delays

FIgURE 5: The velocity errors of agents in TVFT.

numerical simulations are shown to verify the theoretical analysis.

\section{Conflict of Interests}

The authors declare that there is no conflict of interests regarding the publication of this paper.

\section{Acknowledgments}

The authors would like to thank the anonymous reviewers and the Editor $\mathrm{Su}$ for their insightful comments and suggestions. This research is supported by the Chinese Universities Specialized Research Fund for the Doctoral Program (20110185110020), NSFC (61170309, 61104141, and 61301052), the Key Technology R\&D Program of Sichuan Province (2012SZ0159), and 973 Program (2013CB329404).

\section{References}

[1] W. Ren and R. W. Beard, Distributed Consensus in Multi-Vehicle Cooperative Control, Springer, London, UK, 2008.

[2] W. Ren and Y. Cao, Distributed Coordination of Multi-Agent Networks, Springer, London, UK, 2011.

[3] J. Hu and Y. S. Lin, "Consensus control for multi-agent systems with double-integrator dynamics and time delays," IET Control Theory \& Applications, vol. 4, no. 1, pp. 109-118, 2010.

[4] J. Hu and Y. Hong, "Leader-following coordination of multiagent systems with coupling time delays," Physica A, vol. 374, no. 2, pp. 853-863, 2007.

[5] J. Yu and L. Wang, "Group consensus in multi-agent systems with switching topologies and communication delays," Systems \& Control Letters, vol. 59, no. 6, pp. 340-348, 2010.

[6] W. Zhu and D. Cheng, "Leader-following consensus of secondorder agents with multiple time-varying delays," Automatica, vol. 46, no. 12, pp. 1994-1999, 2010.
[7] K. Peng and Y. Yang, "Leader-following consensus problem with a varying-velocity leader and time-varying delays," Physica A, vol. 388, no. 2-3, pp. 193-208, 2009.

[8] Z. Li, Y. Xia, and X. Cao, "Adaptive control of bilateral teleoperation with unsymmetrical time-varying delays," International Journal of Innovative Computing, Information and Control, vol. 9, no. 2, pp. 753-767, 2013.

[9] H. Xia, T. Huang, J. Shao, and J. Yu, "Second-order leaderfollowing consensus of multi-agent systems with time delays," Mathematical Problems in Engineering, vol. 2013, Article ID 505434, 8 pages, 2013.

[10] M. Nguyen and D. Tran, "A combination trust model for multiAgent systems," International Journal of Innovative Computing, Information and Control, vol. 9, no. 6, pp. 2405-2420, 2013.

[11] L. Wu, X. Su, and P. Shi, "Output feedback control of markovian jump repeated scalar nonlinear Systems," IEEE Transactions on Automatic Control, no. 99, 2013.

[12] L. Wu, W. Zheng, and H. Gao, "Dissipativity-based sliding mode control of switched stochastic systems," IEEE Transactions on Automatic Control, vol. 58, no. 3, pp. 785-793, 2013.

[13] T. Balch and R. C. Arkin, "Behavior-based formation control for multirobot teams," IEEE Transactions on Robotics and Automation, vol. 14, no. 6, pp. 926-939, 1998.

[14] M. A. Lewis and K.-H. Tan, "High precision formation control of mobile robots using virtual structures," Autonomous Robots, vol. 4, no. 4, pp. 387-403, 1997.

[15] J. Shao, G. Xie, and L. Wang, "Leader-following formation control of multiple mobile vehicles," IET Control Theory and Applications, vol. 1, no. 2, pp. 545-552, 2007.

[16] F. Xiao, L. Wang, J. Chen, and Y. Gao, "Finite-time formation control for multi-agent systems," Automatica, vol. 45, no. 11, pp. 2605-2611, 2009.

[17] K. Choi, S. J. Yoo, J. B. Park, and Y. H. Choi, "Adaptive formation control in absence of leader's velocity information," IET Control Theory \& Applications, vol. 4, no. 4, pp. 521-528, 2010.

[18] J.-L. Wang and H.-N. Wu, "Leader-following formation control of multi-agent systems under fixed and switching topologies," International Journal of Control, vol. 85, no. 6, pp. 695-705, 2012. 
[19] X. Luo, N. Han, and X. Guan, "Leader-following consensus protocols for formation control of multi-agent network," Journal of Systems Engineering and Electronics, vol. 22, no. 6, pp. 991-997, 2011.

[20] H. Rezaee and F. Abdollahi, "Motion synchronization in unmanned aircrafts formation control with communication delays," Communications in Nonlinear Science and Numerical Simulation, vol. 18, no. 3, pp. 744-756, 2013.

[21] X. Lu, F. Austin, and S. Chen, "Formation control for secondorder multi-agent systems with time-varying delays under directed topology," Communications in Nonlinear Science and Numerical Simulation, vol. 17, no. 3, pp. 1382-1391, 2012.

[22] P. C. Parks and V. Hahn, Stability Theory, Prentice-Hall, Upper Saddle River, NJ, USA, 1992.

[23] J. K. Hale and S. M. Verduyn Lunel, Introduction to FunctionalDifferential Equations, vol. 99 of Applied Mathematical Sciences, Springer, New York, Ny, USA, 1993. 


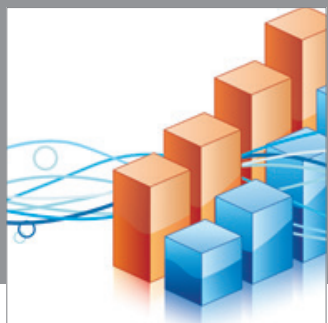

Advances in

Operations Research

mansans

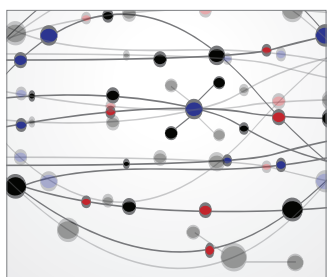

The Scientific World Journal
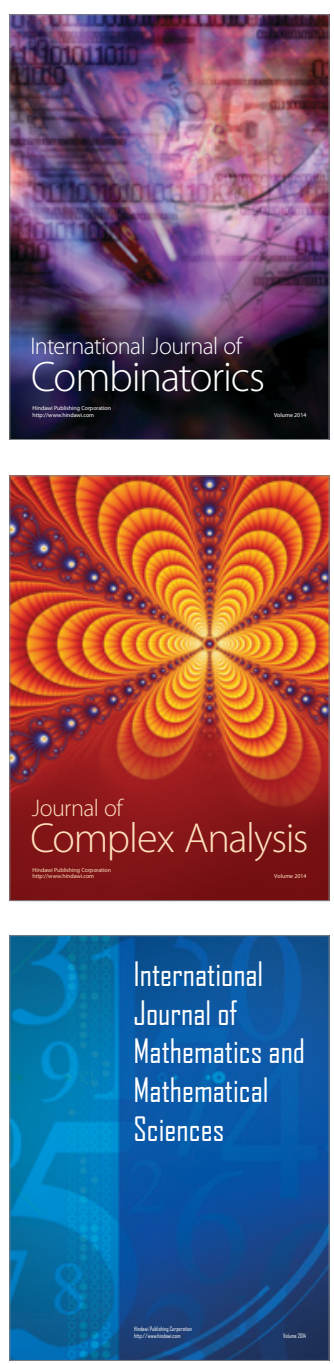
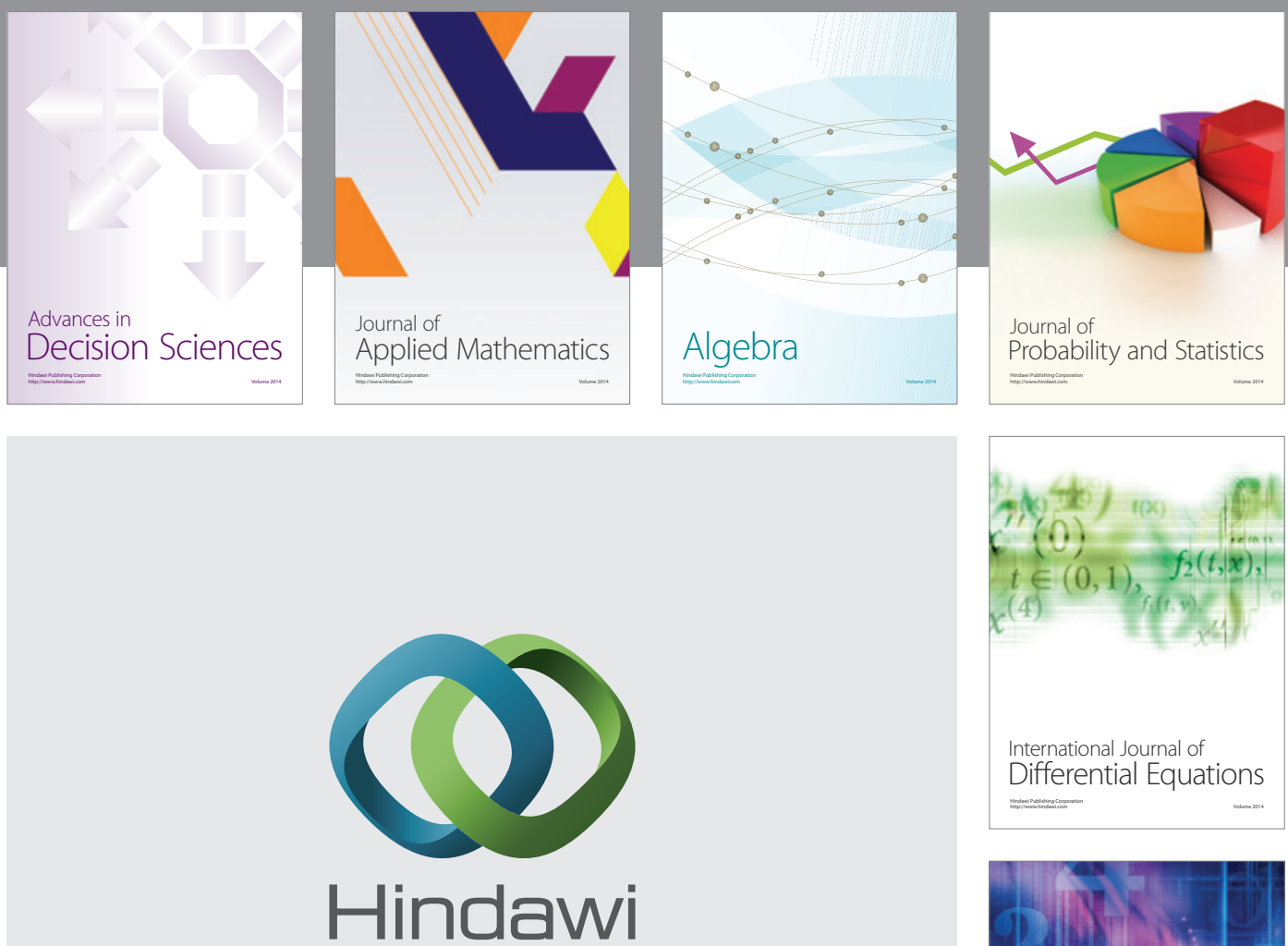

Submit your manuscripts at http://www.hindawi.com
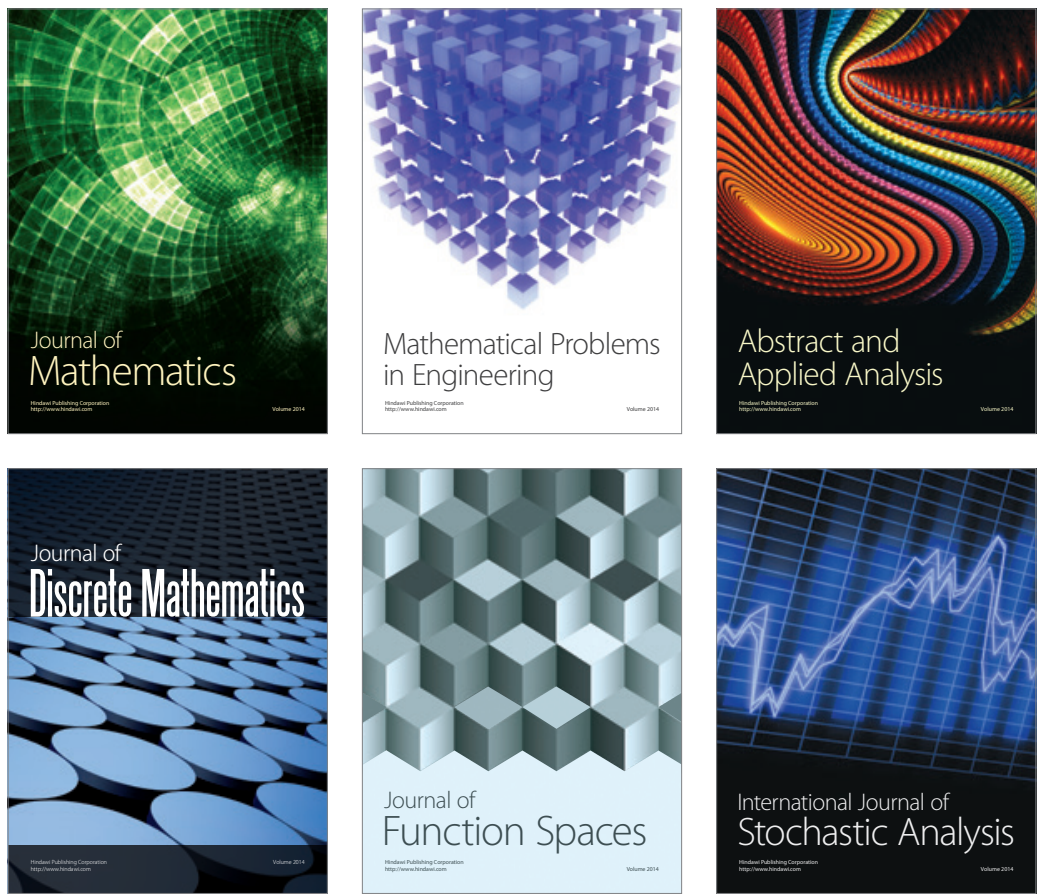

Journal of

Function Spaces

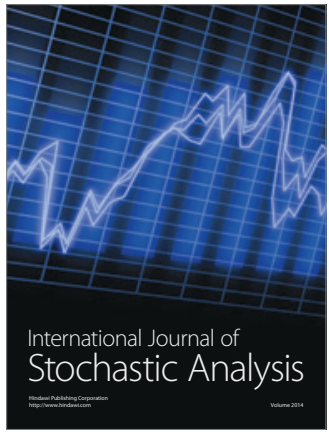

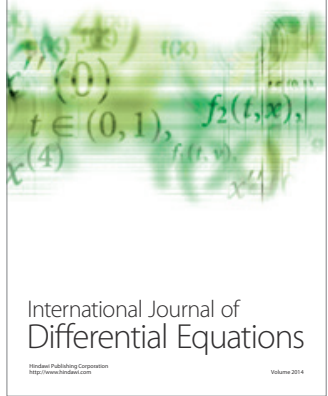
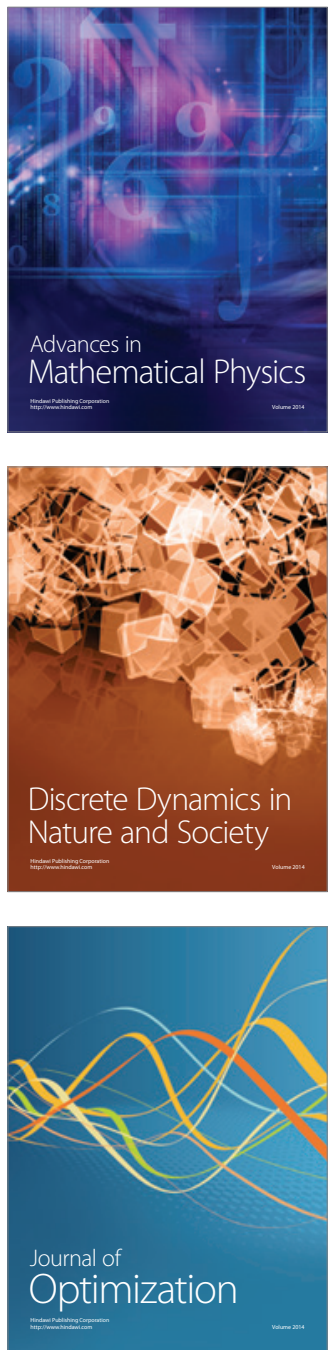